\title{
GEOMETRIC PROPERTIES OF A CLASS OF SUPPORT POINTS OF UNIVALENT FUNCTIONS ${ }^{1}$
}

\author{
BY
}

JOHNNY E. BROWN

\begin{abstract}
Let $S$ denote the set of functions $f(z)$ analytic and univalent in $|z|<1$, normalized by $f(0)=0$ and $f^{\prime}(0)=1$. A function $f$ is a support point of $S$ if there exists a continuous linear functional $L$, nonconstant on $S$, for which $f$ maximizes $\operatorname{Re} L(g), g \in S$. The support points corresponding to the point-evaluation functionals are determined explicitly and are shown to also be extreme points of $S$. New geometric properties of their omitted ares $\Gamma$ are found. In particular, it is shown that for each such support point $\Gamma$ lies entirely in a certain half-strip, $\Gamma$ has monotonic argument, and the angle between radius and tangent vectors increases from zero at infinity to a finite maximum value at the tip of the arc $\Gamma$. Numerical calculations appear to indicate that the known bound $\pi / 4$ for the angle between radius and tangent vectors is actually best possible.
\end{abstract}

Let $H(D)$ denote the space of functions analytic in the unit disk $D$. Let $S$ be the subset of $H(D)$ of functions univalent in $D$ and normalized by the conditions $f(0)=0$ and $f^{\prime}(0)=1$. With the topology of uniform convergence on compact subsets of $D, H(D)$ is a locally convex space and $S$ a compact subset. By the Krein-Milman theorem, $S$ is contained in the closed convex hull of its extreme points [4]. Hence the study of linear functionals on $S$ reduces to their study on the extreme points on $S$. It is therefore an important problem to characterize these extreme points. However, very little is known in this direction. Recently, Brickman [1] used an elementary argument which showed that the extreme points of $S$ map the unit disk onto the complement of a single arc tending to infinity with increasing modulus.

We call $f \in S$ a support point of $S$ if there exists a continuous linear functional $L$ on $H(D)$, not constant on $S$, for which $f$ maximizes $\operatorname{Re} L(g)$, $g \in S$. It is known ([3], [7], [8]) that each support point of $S$ maps the disk onto the complement of a single analytic arc $\Gamma$ with increasing modulus and asymptotic direction at infinity. Furthermore, $\Gamma$ has the property that the angle between radius and tangent vectors never exceeds $\pi / 4$.

It is not known whether each extreme point is a support point, or whether

Presented to the Society, January 24, 1979; received by the editors October 18, 1978.

AMS (MOS) subject classifications (1970). Primary 30A38; Secondary 30A36.

Key words and phrases. Support points, extreme points, univalent functions.

'This paper is part of the author's doctoral thesis written at the University of Michigan under the direction of P. L. Duren. 
each support point is an extreme point for the class $S$. However, it is known [2] that the Koebe function and its rotations belong to both families but do not exhaust either. The purpose of the present paper is to study some other examples and to describe their omitted arcs geometrically. The results may suggest some general properties of support points or extreme points.

Specifically, we study the support points of $S$ corresponding to pointevaluation functionals: $L(g)=g\left(z_{0}\right), z_{0} \in D, z_{0} \neq 0$. These support points turn cut also to be extreme points. It was previously known [10] that for $z_{0}$ off a certain segment of the real axis, these support points do not include any rotation of the Koebe function. We show that the omitted arc $\Gamma$ of each such support point lies in a certain half-strip, has monotonic argument and has an angle between radius and tangent vectors which increases from zero at infinity to a maximum value at the finite tip of the omitted arc. Also, numerical calculations indicate that the bound $\pi / 4$ is best possible: for a certain point $z_{0}$ on the negative real axis, the angle at the tip of the arc approximates $\pi / 4$ to five decimal places.

Our main tool in this investigation is Schiffer's method of boundary variation. In principle, the maximum of $\operatorname{Re} g\left(z_{0}\right)$ can be determined by appealing to Grunsky's result that the region of values of $\log \left(g\left(z_{0}\right) / z_{0}\right)$ is a certain closed circular disk [5]. This approach is rather uninformative since the extremal functions are not identified. Our approach will lead to explicit formulas for the extremal functions.

1. The Schiffer differential equation. Let $f \in S$ be a support point for $L(g)=g\left(z_{0}\right)$. Without loss of generality, we may assume that $\operatorname{Im}\left\{z_{0}\right\}>0$, since the function $\bar{f}$ defined by $\bar{f}(z)=\overline{f(\bar{z})}$ is a support point for $\bar{L}(g)=g\left(\bar{z}_{0}\right)$. Let $\Gamma=C-f(D)$ be the arc omitted by $f$. Following Schiffer [9], we construct a family of neighboring functions $f^{*} \in S$ as follows:

$$
f^{*}(z)=f(z)+\lambda_{\succ}\left[\frac{f(z)^{2}}{w_{0}^{2}\left(f(z)-w_{0}\right)}\right]+O\left(r^{3}\right)
$$

where $w_{0} \in \Gamma$ and $\lambda_{r}=O\left(r^{2}\right)$ as $r$ tends to zero. Letting $B=f\left(z_{0}\right)$ and using the extremality of $f$, we get

$$
\operatorname{Re}\left\{\lambda_{r} s\left(w_{0}\right)+O\left(r^{3}\right)\right\}<0
$$

where $s(w)=B^{2} / w^{2}(B-w)$. By Schiffer's fundamental lemma [9] we conclude that $\Gamma$ is an analytic arc satisfying

$$
\frac{B^{2}}{w^{2}(B-w)}\left[\frac{d w}{d t}\right]^{2}>0, \quad w=w(t) .
$$


If we parameterize $\Gamma$ by $w(t)=f\left(e^{i t}\right)$, then (1) becomes

$$
\left[\frac{z f^{\prime}(z)}{f(z)}\right]^{2} \frac{B^{2}}{[f(z)-B]}>0, \quad|z|=1 .
$$

For $|z|<1$, let $F(z)$ denote the left-hand side of (2). Clearly, $F(z)$ is analytic for $|z|<1$ except for a simple pole at $z_{0}$, and $F(z)$ has a zero of order two at a point $e^{i \alpha}$ corresponding to the finite tip of the arc, where $f^{\prime}\left(e^{i \alpha}\right)=0$. By (2), $F(z)>0$ on $|z|=1$. Hence we may apply the Schwarz reflection principle to conclude that $F(z)$ is actually a rational function of the form

$$
F(z)=\frac{A\left(z-e^{i \alpha}\right)^{2}}{\left(z-z_{0}\right)\left(1-\bar{z}_{0} z\right)},
$$

where $A$ is a constant. If $e^{i \theta} \neq e^{i \alpha}$, we conclude from the representation (3) that

$$
0<F\left(e^{i \theta}\right)=\frac{-4 A e^{i \alpha} \sin ^{2}((\theta-\alpha) / 2)}{\left|e^{i \theta}-z_{0}\right|^{2}} .
$$

This yields $A e^{i \alpha}<0$. If we now equate the two representations (2) and (3) for $F(z)$ and let $z$ tend to zero, we get that $A e^{i \alpha}=z_{0} B e^{-i \alpha}$. Hence,

$$
e^{i \alpha}=-z_{0} B /\left|z_{0} B\right|, \quad B=f\left(z_{0}\right) .
$$

Equating the two representations again and using (4), we obtain

$$
\left[\frac{z f^{\prime}(z)}{f(z)}\right]^{2} \frac{B}{[B-f(z)]}=\frac{\left(1-e^{-i \alpha} z\right)^{2}}{\left(1-z / z_{0}\right)\left(1-\bar{z}_{0} z\right)}, \quad B=f\left(z_{0}\right) .
$$

In general the Schiffer differential equation is difficult to integrate and may involve several unknown parameters. However, (5) may be integrated to obtain a closed form expression for $f(z)$ and the unknown parameter can be determined.

Let $w=f(z)$ and $P(z)=\left(1-z / z_{0}\right)\left(1-\bar{z}_{0} z\right)$ then (5) may be integrated to obtain

$$
\int_{B}^{w} \frac{d w}{w \sqrt{1-w / B}}=\int_{z_{0}}^{z} \frac{\left(1-e^{-i \alpha} z\right)}{\sqrt{P(z)}} \frac{d z}{z},
$$

where $|z|<1$ and we have chosen the branch of the square root that is +1 when $z=0$. If we now let $\left|z_{0}\right|=r$ and let

$$
M(z)=\left[\frac{\left(1-r^{2} z / z_{0}\right)-r \sqrt{P(z)}}{\left(1-r^{2} z / z_{0}\right)+r \sqrt{P(z)}}\right]
$$

and

$$
Q(z)=\left[2 \sqrt{P(z)}+2-\left(1+r^{2}\right) z / z_{0}\right]
$$


then using (4) we see that equation (6) takes the form

$$
I=I_{1}+\frac{|B|}{B} I_{2}
$$

where

$$
\begin{aligned}
& I=\int_{B}^{w} \frac{d w}{w \sqrt{1-w / B}}=\log \left[\frac{1-\sqrt{1-w / B}}{1+\sqrt{1-w / B}}\right], \\
& I_{1}=\int_{z_{0}}^{z} \frac{d z}{z \sqrt{P(z)}}=-\log \left[\frac{Q(z)}{1-r^{2}} \cdot \frac{z_{0}}{z}\right] \\
& I_{2}=\frac{r}{z_{0}} \int_{z_{0}}^{z} \frac{d z}{\sqrt{P(z)}}=\log M(z) .
\end{aligned}
$$

After a straightforward calculation we find that

$$
f(z)=4 B \cdot V(z) /[1+V(z)]^{2}
$$

where $V(z)=\left(z / z_{0}\right)\left(1-r^{2}\right) M(z)^{e} / Q(z)$ and $\varepsilon=|B| / B$. Hence we have determined the support point in terms of $z_{0}$ and $B=f\left(z_{0}\right)$.

Adding $-\log z$ to both sides of (7) and letting $z$ tend to zero, we get

$$
\log \frac{z_{0}}{B}=\log \left(1-r^{2}\right)+\frac{|B|}{B} \log \left(\frac{1-r}{1+r}\right) \text {. }
$$

Letting $B=|B| e^{i \theta_{0}}$ and taking the real part of (9) gives an expression for $|B|$ and the imaginary part of (9) may be written as

$$
\theta_{0}+R \sin \theta_{0}=\arg z_{0}
$$

where $R=\log ((1+r) /(1-r))$. Thus, the maximum of $\operatorname{Re} g\left(z_{0}\right), g \in S$, is given by

$$
\operatorname{Re}\left\{f\left(z_{0}\right)\right\}=\left(\cos \theta_{0}\right) \frac{r}{1-r^{2}}\left(\frac{1+r}{1-r}\right)^{\cos \theta_{0}},
$$

where $\theta_{0}=\arg f\left(z_{0}\right)$.

Equation (10) has the form

$$
R \sin \theta_{0}=\arg z_{0}-\theta_{0}
$$

where $R=\log ((1+r) /(1-r))$. If $0<\arg z_{0}<\pi$, this equation has a unique solution $\theta_{0}, 0<\theta_{0}<\pi$. If $\arg z_{0}=0$, then $\theta_{0}=0$ is the only solution. If $\arg z_{0}=\pi$, then $\theta_{0}=\pi$ is the unique solution if $0<R<1$; while if $R>1$ there are two solutions, one being $\theta_{0}=\pi$. In this last case $\theta_{0}=\pi$ is an extraneous solution, since it gives a value of $B$ with smaller real part. Thus $\theta_{0}=\pi$ is the unique solution if and only if $(1-e) /(1+e)<z_{0}<0$. In view of (11), this shows that the Koebe function is the unique extremal function if $z_{0}$ is real and $(1-e) /(1+e)<z_{0}<1, z_{0} \neq 0$. (This result is stated in Schober [10, p. 84].) Hence if $z_{0} \notin[(1-e) /(1+e), 1)$ then $\operatorname{Im} B>0$ and 
there is a unique extremal function which is not the Koebe funtion or a rotation. Indeed, if $z_{0} \notin[(1-e) /(1+e), 1)$ and if $f(z)=z /(1-x z)^{2},|x|=$ 1, were to maximize $\operatorname{Re} g\left(z_{0}\right)$, then it would follow from (2) that

$$
\arg B^{2}=\arg \left(B-f\left(e^{i \theta}\right)\right)
$$

for $e^{i \theta} \neq e^{i \alpha}$, which would imply $\operatorname{Im} B=0$. Because of the uniqueness, (8) actually defines an extreme point of $S$, by the Krein-Milman theorem.

2. Geometric properties of the omitted arc. Let $f \in S$ be a support point for $L(g)=g\left(z_{0}\right)$. We continue to assume that $\operatorname{Im} z_{0}>0$. We have observed that if $\operatorname{Im} z_{0}>0$ then $\operatorname{Im} f\left(z_{0}\right)>0$. It was also shown that if $\operatorname{Im} f\left(z_{0}\right)=0$ then the Koebe function is the only solution. Thus, in what follows, we may assume that $\operatorname{Im} z_{0}>0, z_{0} \notin[(1-e) /(1+e), 1)$ and $\theta_{0}=\arg f\left(z_{0}\right)$ lies in $(0, \pi)$.

Let the omitted arc $\Gamma=C-f(D)$ be parameterized by $w=w(t)$ for $0<t<T<\infty, w^{\prime}(t) \neq 0$ and $w_{0}=w(0)$ is the finite tip of the arc. Let $\Theta(t)=\arg w(t)$ and let $A(t)=\arg \left(w(t) / w^{\prime}(t)\right)$, for $0<t<T$, be the angle between the radius and tangent vectors. As above, we let $B=f\left(z_{0}\right)$ with $\theta_{0}=\arg B$ lying in $(0, \pi)$. We shall prove the following theorems.

Throrem 1. $A(t)$ decreases to zero as $t$ increases to $T$. Thus $0<A(t)<A(0)$, for $0<t<T$, where $A(0)=\lim _{t \rightarrow 0} A(t)$.

THEOREM 2. $\Theta(t)$ is a decreasing function of $t$. Furthermore, each ray from the origin intersects $\Gamma$ in at most one point.

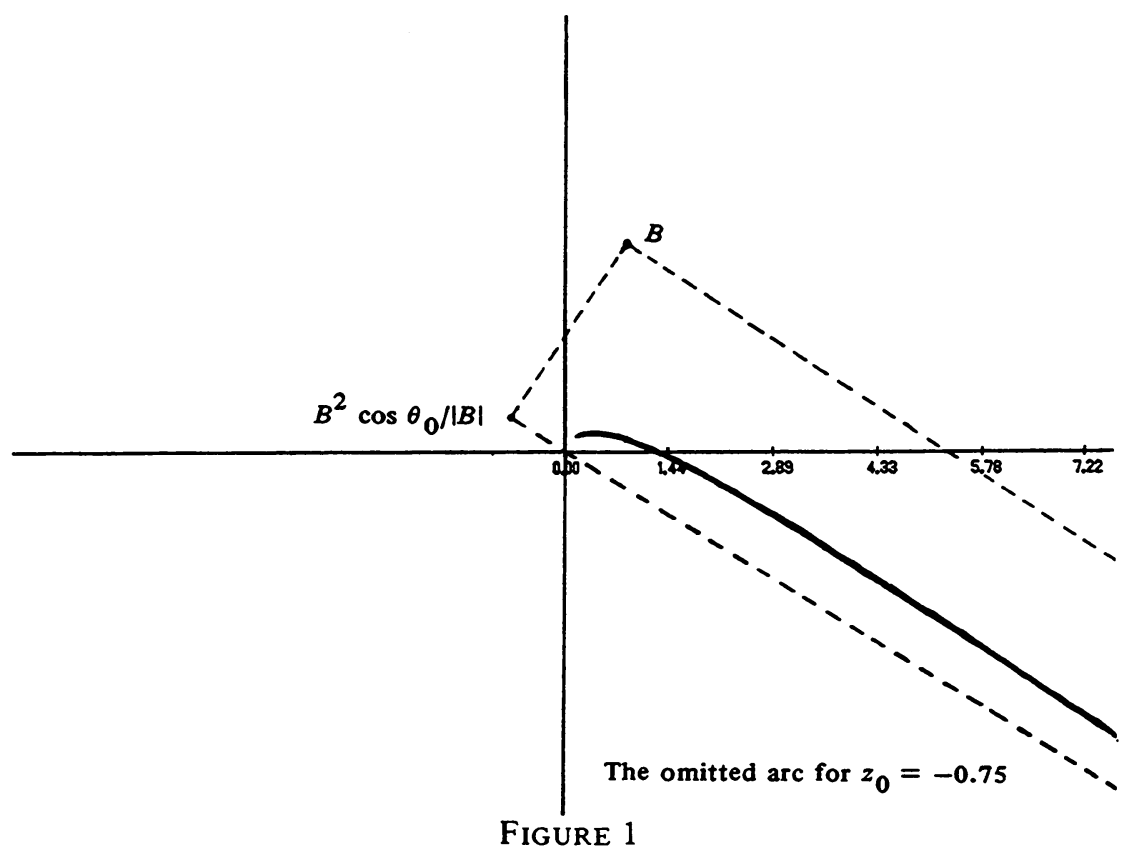


THEOREM 3. $\Gamma$ lies entirely in the half-strip with vertices $B$ and $B^{2} \cos \theta_{0} /|B|$, bounded by the segment joining these two points, the half-line through the origin terminating at the second point, and the parallel half-line terminating at B. (See Figure 1.)

Before embarking upon the proofs of these theorems, we first derive an explicit formula for $w(t)$ as a certain logarithmic spiral composed with the Koebe function. The parameterization $w=w(t)$ may be chosen so that (1) becomes

$$
\frac{B^{2}}{B-w(t)}\left[\frac{w^{\prime}(t)}{w(t)}\right]^{2}=1, \quad 0<t<T .
$$

From this equation we get

$$
\frac{1}{w \sqrt{1-w / B}} \frac{d w}{d t}=\frac{1}{\sqrt{B}}, \quad 0<t<T,
$$

where we have chosen the branch of $\sqrt{B}$ for which $\arg \sqrt{B}=\theta_{0} / 2$. If we integrate this equation we obtain after a calculation

$$
w(t)=-4 B k(s(t)), \quad 0<t<T,
$$

where

$$
s(t)=c e^{t / V B}, \quad c=\frac{\sqrt{1-w_{0} / B}-1}{\sqrt{1-w_{0} / B}+1}
$$

and $k(z)=z /(1-z)^{2}$ is the usual Koebe function. Hence $\Gamma$ is the image under the Koebe function of part of a logarithmic spiral which we will call $\gamma$.

We must investigate $\gamma$ more closely. Since $\Gamma$ is unbounded we see that $s(T)=1$. We may therefore rewrite (14) in the form $s(t)=r(t) e^{i \psi(t)}$, where

$$
r(t)=\exp \left[\operatorname{Re}\left(\frac{t-T}{\sqrt{B}}\right)\right]
$$

and

$$
\psi(t)=\operatorname{Im}\left(\frac{t-T}{\sqrt{B}}\right) .
$$

Observe first that since $\sqrt{B}$ lies in the first quadrant that $r(t)$ increases to 1 and $\psi(t)$ decreases to 0 . Secondly, the following lemma shows that $\gamma$ does not wind around the origin.

LEMMA 1. If $\psi(t)$ is defined by (16) and $0<\theta_{0}<\pi$ then $0<\psi(t)<\pi$ for $0<t<T$. Hence $\operatorname{Im}\{s(t)\}>0$ for $0<t<T$.

The proof of this lemma depends on two preliminary technical lemmas: 
LEMMA 2. If $0<\theta<\pi / 2$ then

$$
\begin{aligned}
d(\theta)= & -2(\theta-\sin \theta)(\sin \theta+(\pi-\theta) \cos \theta) \\
& +\sin \theta(1-\cos \theta)(2 \sin \theta-\theta)<0 .
\end{aligned}
$$

LEMMA 3. If $B=|B| e^{i \theta_{0}}$ and $0<\theta_{0}<\pi / 2$ then

$$
4|B| k\left(e^{-\pi \cot \left(\theta_{0} / 2\right)}\right)<\frac{1}{4} \text {. }
$$

We show first that Lemma 1 may be deduced from Lemmas 2 and 3. The proofs of Lemmas 2 and 3 follow afterwards.

Proof of Lemma 1. This proof is separated into two cases.

Case 1. $\pi / 2<\theta_{0}<\pi$. Assume there exists a $t_{1}$ with $0<t_{1}<T$ satisfying $\psi\left(t_{1}\right)=\pi$. Then from (15) and (16), $s\left(t_{1}\right)=-x$ for some $0<x<1$. Now since $w(t)=-4 B k(s(t))$, taking the logarithmic derivative and using (14) we get

$$
\frac{w^{\prime}(t)}{w(t)}=\frac{1+s(t)}{1-s(t)} \cdot \frac{1}{\sqrt{B}} .
$$

Since $\pi / 2<\theta_{0}<\pi$, we can conclude that

$$
\operatorname{Re}\left\{\left(\frac{w^{\prime}\left(t_{1}\right)}{w\left(t_{1}\right)}\right)^{2}\right\}=\left(\frac{1-x}{1+x}\right)^{2} \cdot \operatorname{Re}\left\{\frac{1}{B}\right\}<0 .
$$

This contradicts the ( $\pi / 4)$-property [3].

Case 2. $0<\theta_{0}<\pi / 2$. As a consequence of Lemma 3 we can conclude that

$$
|c|>e^{-\pi \cot \left(\theta_{0} / 2\right)} \text {. }
$$

Indeed, if $|c|<e^{-\pi \cot \left(\theta_{0} / 2\right)}$, then since $w_{0}=-4 B k(c)$, we get

$$
\left|w_{0}\right|=4|B||k(c)|<4|B| k(|c|)<4|B| k\left(e^{-\pi \cot \left(\theta_{0} / 2\right)}\right) \text {. }
$$

In view of Lemma 3 we see that $\left|w_{0}\right|<\frac{1}{4}$ and this contradicts the Koebe $\frac{1}{4}$-theorem [10]. Hence (17) holds. From (15) we see that $|c|=\exp \{\operatorname{Re}(-T / \sqrt{B})\}$ and from (17) we may conclude that

$$
\operatorname{Re}(\mathrm{T} / \sqrt{\mathrm{B}}) \cdot \tan \left(\theta_{0} / 2\right)<\pi \text {. }
$$

Now using the identity that $\operatorname{Im}(-T / \sqrt{B})=\operatorname{Re}(T / \sqrt{B}) \cdot \tan \left(\theta_{0} / 2\right)$ we get

$$
0<\psi(t)=\operatorname{Im}\left(\frac{t-T}{\sqrt{B}}\right)<\operatorname{Im}\left(\frac{-T}{\sqrt{B}}\right)<\pi
$$

for $0<t<T$. The proof of the lemma is complete.

Proof of Lemma 2. Note that for $0<\theta<\pi / 2$, the following inequality holds:

$$
\sin \theta(1-\cos \theta)<3(\theta-\sin \theta) .
$$

This is true since the difference of these two functions vanishes at $\theta=0$ and is a decreasing function of $\theta$. From (18) we see that 


$$
d(\theta)<(\theta-\sin \theta) g(\theta)
$$

where

$$
g(\theta)=4 \sin \theta-3 \theta-2(\pi-\theta) \cos \theta .
$$

Since $\theta-\sin \theta>0$, it suffices to show that $g(\theta)<0$ for $0<\theta<\pi / 2$.

Case $1.0<\theta<\pi / 4$. By using the fact that $\theta-\sin \theta>0$, we get

$$
g(\theta)=\sin \theta-3(\theta-\sin \theta)-2(\pi-\theta) \cos \theta<g_{1}(\theta),
$$

where $g_{1}(\theta)=\sin \theta-2(\pi-\theta) \cos \theta$. Now observe that $g_{1}(\pi / 4)=$ $(\sqrt{2} / 2)(1-(3 \pi / 2))<0$ and $g_{1}^{\prime}(\theta)>0$. Hence $g_{1}(\theta)<0$ and so $g(\theta)<0$.

Case 2. $\pi / 4<\theta<\pi / 2$. We see that

$$
g^{\prime}(\theta)=6 \cos \theta-3+2(\pi-\theta) \sin \theta
$$

and

$$
g^{\prime \prime}(\theta)=-8 \sin \theta+2(\pi-\theta) \cos \theta .
$$

Now $g^{\prime \prime}$ is decreasing and we see that for $\pi / 4<\theta<\pi / 2$,

$$
g^{\prime \prime}(\theta)<g^{\prime \prime}\left(\frac{\pi}{4}\right)=\frac{\sqrt{2}}{2}\left(\frac{3 \pi}{2}-8\right)<0 .
$$

Hence $g^{\prime}$ is also decreasing in this interval so that

$$
g^{\prime}(\theta)>g^{\prime}(\pi / 2)=\pi-3>0 .
$$

This shows that $g$ is increasing for $\pi / 4<\theta<\pi / 2$ and that

$$
g(\theta)<g\left(\frac{\pi}{2}\right)=4-\frac{3 \pi}{2}<0 .
$$

The proof of the lemma is complete.

Proof of LemMa 3. Recall that if $z_{0}=r e^{i x_{0}}, 0<r<1,0<x_{0}<\pi$ then from (9) we have

$$
|B|=\frac{r}{1-r^{2}}\left(\frac{1+r}{1-r}\right)^{\cos \theta_{0}}
$$

where $\theta_{0}+R \sin \theta_{0}=x_{0}$ and $R=\log ((1+r) /(1-r))$. A brief calculation shows that

$$
4|B| k\left(e^{-\pi \cot \left(\theta_{0} / 2\right)}\right)=e^{R \cos \theta_{0}}\left(e^{R}-e^{-R}\right) k\left(e^{-\pi \cot \left(\theta_{0} / 2\right)}\right) .
$$

Now since $k\left(e^{-\pi \cot \left(\theta_{0} / 2\right)}\right)<1.093 e^{-\pi \cot \left(\theta_{0} / 2\right)}$, the lemma will follow if we show that

$$
\exp \left[R \cos \theta_{0}-\pi \cot \left(\theta_{0} / 2\right)\right]\left(e^{R}-e^{-R}\right)(1.093)<\frac{1}{4} .
$$

For a fixed value of $\theta_{0}, 0<\theta_{0}<\pi / 2$, the left-hand side of (19) is maximal when $R$ is maximal. Now since $\theta_{0}+R \sin \theta_{0}=x_{0}$ and $0<x_{0}<\pi$, the left-hand side is maximal precisely when $R=\left(\pi-\theta_{0}\right) / \sin \theta_{0}$. Thus, inequality (19) will follow if we show that, for any $\theta, 0<\theta<\pi / 2$, 


$$
\exp \left[\frac{\pi-\theta}{\tan \theta}-\pi \cot \frac{\theta}{2}\right]\left(e^{((\pi-\theta) / \sin \theta)}-e^{-((\pi-\theta) / \sin \theta)}\right)(1.093)<\frac{1}{4} .
$$

Let $G(\theta)$ denote the left-hand side of $(20)$. Observe that $G(\pi / 2)<0.22<\frac{1}{4}$. We will show that $G$ is an increasing function. For convenience, set

$$
\begin{aligned}
& p(\theta)=\frac{1.093}{\sin ^{2} \theta} \cdot \exp \left[\frac{\pi-\theta}{\tan \theta}-\pi \cot \frac{\theta}{2}-\frac{\pi-\theta}{\sin \theta}\right] \\
& m(\theta)=\exp \left[2\left(\frac{\pi-\theta}{\sin \theta}\right)\right] \cdot(\theta-\sin \theta)(1+\cos \theta)
\end{aligned}
$$

and

$$
h(\theta)=2 \pi \cos \theta+(\theta+\sin \theta)(1-\cos \theta) .
$$

It can be shown that if $0<\theta<\pi / 2$ then $p(\theta)>0$ and $h(\theta)<2 \pi$. A calculation shows that

$$
m^{\prime}(\theta)=\frac{\exp [2((\pi-\theta) / \sin \theta)]}{1-\cos \theta} \cdot d(\theta),
$$

where $d(\theta)$ is defined as in Lemma 2. Consequently, by Lemma 2, $m(\theta)$ is a decreasing function. Now simply observe the following:

$$
\begin{aligned}
G^{\prime}(\theta) & =p(\theta)[m(\theta)-h(\theta)] \\
& >p(\theta)[m(\pi / 2)-2 \pi]=p(\theta)\left[e^{\pi}(\pi / 2-1)-2 \pi\right]>0 .
\end{aligned}
$$

Hence, $G(\theta)<G(\pi / 2)<\frac{1}{4}$ for $0<\theta<\pi / 2$. The proof of the lemma is complete.

We observed from (15) that $r(t)$ is increasing with $t$ and hence we may introduce $r$ as the independent variable and express $\gamma$ by the equation

$$
r^{\tan \left(\theta_{0} / 2\right)} e^{\psi}=1 \text {, }
$$

where $0<r_{0}<r<1$ and $0<\psi<\psi_{0}<\pi$ with $c=r_{0} e^{i \psi_{0}}$. An easy calculation shows that, for $0<t<T$,

$$
A(t)=\arg \left(\frac{w(t)}{w^{\prime}(t)}\right)=\frac{\theta_{0}}{2}-\tan ^{-1}\left[\frac{2 r(t)}{1-r(t)^{2}} \sin \psi(t)\right],
$$

where $r(t)$ and $\psi(t)$ satisfy (15) and (16). Consider the function $a(r)$ defined as follows:

$$
a(r)=\frac{\theta_{0}}{2}-\tan ^{-1}\left[\frac{2 r}{1-r^{2}} \sin \psi\right],
$$

where $r$ and $\psi$ are related by (21). From the above we get $A(t)=a(r(t))$. Hence $a(r)$, subject to $(21)$, measures the angle between radius and tangent vectors in terms of the spiral $\gamma$. The behavior of $a(r)$ plays a central role in the proofs of the theorems. We shall also use the following lemma. 
LEMMA 4. Fix $\alpha, 0<\alpha<\infty$, and let $\rho=e^{-\pi / \alpha}$. Let $r$ and $x$ satisfy the equation $r^{\alpha} e^{x}=1$, where $0<\rho<r<1$ and $0<x<\pi$. Then

$$
2 r \sin x<\alpha\left(1-r^{2}\right) \text {. }
$$

Equality occurs if and only if $r=1$.

ProOF. Since $r=e^{-x / \alpha}$ we see that

$$
2 r \sin x-\alpha\left(1-r^{2}\right)=2 e^{-x / \alpha} \Phi(x),
$$

where $\Phi(x)=\sin x-\alpha \sinh (x / \alpha)$. Clearly $\Phi(0)=0$ and $\Phi^{\prime}(x)<0$ for $0<$ $x<\pi$. Hence $\Phi(x)<0$ for $0<x<\pi$. The proof of the lemma is complete.

PROOF OF THEOREM 1. We remarked previously that $A(r)=a(r(t))$ where $a(r)=\theta_{0} / 2-\tan ^{-1} g(r)$, with $g(r)=\left(2 r /\left(1-r^{2}\right)\right) \sin \psi$ and $r$ and $\psi$ are related by (21). With $x=\psi$ and $\alpha=\tan \left(\theta_{0} / 2\right)$, we conclude from Lemma 4 that $g(r)<\tan \left(\theta_{0} / 2\right), \rho<r_{0}<r<1$. Hence $a(r)>0$ for $\rho<r_{0}<r<1$. It remains to show that $g(r)$ is an increasing function.

A calculation shows that $g^{\prime}(r)=p H(r)$, where

$$
H(r)=\left[\frac{1+r^{2}}{1-r^{2}}-\frac{\tan \left(\theta_{0} / 2\right)}{\tan \psi}\right]
$$

and

$$
p=\frac{2}{1-r^{2}} \sin \psi>0
$$

with $r$ and $\psi$ related by (21).

We find by l'Hôpital's rule that $H(r)$ tends to zero as $r$ tends to 1. Also,

$$
H^{\prime}(r)=q\left[2 r \sin \psi-\tan \left(\theta_{0} / 2\right)\left(1-r^{2}\right)\right]
$$

where

$$
q=\frac{1}{r\left(1-r^{2}\right) \sin \psi}\left[\frac{2 r}{1-r^{2}} \sin \psi+\frac{\tan \left(\theta_{0} / 2\right)}{\sin \psi}\right]>0 .
$$

Another application of Lemma 4 shows that $H^{\prime}(r)<0$, so $H(r)>0$ for $\rho<r_{0}<r<1$. Hence $g^{\prime}(r)>0$, and $g(r)$ is increasing. This proves that $a(r)$ is decreasing; hence $A(t)$ is decreasing.

Proof of Theorem 2. Combining the previous theorem with the $\pi / 4$ property, we get $0<A(t)<\pi / 4$ for $0<t<T$. This implies that $\theta^{\prime}(t)=$ $\operatorname{Im}\left(w^{\prime}(t) / w(t)\right)<0$ for $0<t<T$. Hence $\theta(t)$ is decreasing.

We have already observed that $\operatorname{Im} s(t)>0$. Since the Koebe function preserves the upper half-plane, we can conclude from (13) that $\operatorname{Im}(\bar{B} w(t))<$ 0 . In particular, $\Gamma$ lies in a half-plane and does not wind around the origin. Thus, each ray from the origin intersects $\Gamma$ at most once.

Proof of Theorem 3. Brickman and Wilken [3] have shown that $\Gamma$ is asymptotic to a fixed line at infinity given by $d_{-1} / t+d_{0}, 0<t<\delta$, where 
$d_{-1}=-4 L\left(f^{2}\right)$ and $d_{0}=L\left(f^{3}\right) / 3 L\left(f^{2}\right)$. For our point-evaluation functional, the asymptotic line $l$ is given by $l:-\tau B^{2}+B / 3,4 / \delta<\tau<\infty$. By the $\pi / 4$-property, $|A(t)|<\pi / 4$ for $0<t<T$. In view of (12) we see that $\left|\arg \vec{B}^{2}(B-w(t))\right|<\pi / 2$. Geometrically this says that $\Gamma$ lies in the half-plane bounded by the line through the points $B$ and $B^{2} \cos \theta_{0} /|B|$, containing the line $l$.

Using Theorem 1 we may conclude further that

$$
-\pi / 2<\arg \bar{B}^{2}(B-w(t))<0,
$$

which says that $\Gamma$ lies in the quarter-plane which contains $l$. If $\Gamma$ crosses the line $l^{\prime}:-B^{2} \tau^{\prime}, 0<\tau^{\prime}<\infty$, then, in view of Theorem $2, \Gamma$ cannot approach the line $l$ and a contradiction arises. Hence $\Gamma$ must lie in the indicated half-strip.

3. Numerical calculations. It is known that the omitted arc $\Gamma$ of each support point of $S$ has the $\pi / 4$-property: the angle between radius vector and tangent vector is at most $\pi / 4$. It is an open question whether $\pi / 4$ can be replaced by a smaller bound. Our calculations indicate, however, that even for the support points associated with point-evaluation functionals, $\pi / 4$ is best possible. Specifically, we have used the explicit formulas (9), (10) and (13) for the support point $f$ and its omitted arc $\Gamma$ to show that for $z_{0}=$ -0.56175 , the angle at the tip is $A(0)=0.785393775 \cdots$. Since $\pi / 4=$ $0.78539816 \cdots$, this is a strong indication that $\pi / 4$ is best possible.

Finally, we can make an interesting remark related to recent work of Kirwan and Pell [6]. They showed that the second coefficient of a support point of $S$ always has modulus $\left|a_{2}\right|>\sqrt{2}$. In an effort to see whether $\sqrt{2}$ is best possible, they found that for a certain $z_{0}$ in the interval $-1<z_{0}<0$, $\operatorname{Re} g\left(z_{0}\right)$ is maximized by a function with $1.77<\left|a_{2}\right|<1.774$. Comparing the coefficient of $z$ on both sides of equation (5), we find

$$
a_{2}=\frac{-1}{2 B}+\frac{\left(1+r^{2}\right)}{2 z_{0}}+\frac{|B|}{B} \frac{r}{z_{0}} \text {. }
$$

Our numerical computations indicate that $\left|a_{2}\right|$ is minimal when $z_{0}$ is real and negative, but we have been unable to find a proof. For $z_{0}=-0.75$ we find $\left|a_{2}\right|=1.7737 \cdots$, a result similar to that of Kirwan and Pell.

The author wishes to thank the referee for his helpful comments and Professor Peter Duren for his guidance and inspiration.

\section{REFERENCES}

[1] L. Brickman, Extreme points of the set of univalent functions, Bull. Amer. Math. Soc. 76 (1970), 372-374.

[2] L. Brickman, T. H. MacGregor and D. R. Wilken, Convex hulls of some classical families of univalent functions, Trans. Amer. Math. Soc. 156 (1971), 91-107. 
[3] L. Brickman and D. R. Wilken, Support points of the set of univalent functions, Proc. Amer. Math. Soc. 42 (1974), 523-528.

[4] N. Dunford and J. Schwartz, Linear operators, Part I, Interscience, New York, 1958.

[5] H. Grunsky, Neue Abschätzungen zur konformen Abbildung ein-und mehrfach zusammenhängender Bereiche, Schr. Math. Seminar Univ. Berlin 1 (1932), 93-140.

[6] W. E. Kirwan and R. Pell, Extremal properties of a class of slit conformal mappings, Michigan Math. J. (to appear).

[7] A. Pfluger, Linear extremalprobleme bei schlichten funktionen, Ann. Acad. Sci. Fenn. Ser. A I, No. 489 (1971), 32 pp.

[8] A. C. Schaeffer and D. C. Spencer, Coefficient regions for Schlicht functions, Amer. Math. Soc. Collog. Publ., no. 35, Amer. Math. Soc., Providence, R. I., 1950.

[9] M. Schiffer, A method of variation within the family of simple functions, Proc. London Math. Soc. (2) 44 (1938), $432-449$.

[10] G. Schober, Univalent functions-selected topics, Lecture Notes in Math., vol. 478, SpringerVerlag, Berlin and New York, 1975.

Departagnt of Matheantics, University of Michionn, Ann Arbor, Michignn 48109 